Querverweisen behält Bauerkämper die Singularität des NS-Massenmordes an den Juden im Blick. Die Gesamtargumentation ist stets ausgewogen.

\title{
Westemeier, Jens (Hrsg.): ,So war der deutsche Landser ...“. Das populäre Bild der Wehrmacht (Krieg in der Geschichte, Bd. 101), 361 S., Schöningh, Paderborn 2019.
}

\section{Werner Bührer}

Angenommen: 6. August 2021 / Online publiziert: 26. August 2021

(C) Der/die Autor(en) 2021

Am Ende seiner das breite thematische Spektrum des Bandes präzise zusammenfassenden Einleitung stellt der Herausgeber resigniert fest, dass auch 75 Jahre nach Kriegsende , die Legende von der ,sauberen“ Wehrmacht“ und den deutschen „,Supersoldaten" weiterlebt" (S. 26). Nach der Lektüre der 18 überwiegend von Historikern und Historikerinnen verfassten Beiträge kann man dieses Gefühl durchaus nachvollziehen: zahlreiche einschlägige wissenschaftliche Studien und Ausstellungen konnten die Wehrmachtsapologie nicht stoppen - im Gegenteil, mit dem Internet steht ein neues, massenwirksames Medium für ,postfaktische“ Diskurse über das deutsche Militär als „Kristallisationspunkt für ,Anständigkeit“, ,Ritterlichkeit“, ,Heldentum“ und ,Modernität““ zur Verfügung, so Karsten Wilke in seinem Beitrag (S. 329). Anlass zum Pessimismus liefert auch ein anderer Befund: Leser der kriegsund NS-verherrlichenden „Landser“-Hefte - die Reihe wurde zwar 2013 eingestellt, doch werden die Hefte noch immer ,,auf Flohmärkten und Internetplattformen kartonweise zum Kauf angeboten“ - waren laut Matías Martinez von Anfang an nämlich „weniger Kriegsveteranen als vor allem männliche Jugendliche“ (S. 102).

Der Band geht zurück auf einen im Juli 2016 vom „Arbeitskreis Militärgeschichte“ an der Internationalen Jugendbibliothek in München ausgerichteten Workshop. Er präsentiert ebenso umfassende wie differenzierte und detaillierte Einblicke in das Geflecht der Akteure, Medien und Motive, die zum populären Bild einer ,,ehrenvollen“, ,unpolitischen“ und ,effektiven“Wehrmacht (Jens Westemeier, S. 5) beitragen. Zunächst waren es ehemalige Wehrmachtsoffiziere, allen voran Generaloberst Franz

Werner Bührer $(\triangle)$

Technische Universität München, München, Deutschland

E-Mail: Werner.Buehrer@tum.de 
Halder, der von 1938 bis 1942 als Chef des Generalstabs des Heeres eine ,,maßgebliche Rolle bei der Planung und Verwirklichung des nationalsozialistischen Angriffs-, Eroberungs- und Vernichtungskriegs" gespielt hatte, die als Architekten der Legende von der „sauberen“ Wehrmacht in Erscheinung traten (Esther-Julia Howell, S. 42). Seit Herbst 1946 stieg Halder rasch zu einem der ,wichtigsten deutschen Mitarbeiter“ der „Historical Division“ der U.S. Army auf, die vor dem Hintergrund der Verschärfung des Kalten Krieges vor allem an „Informationen über die deutschen Operationen gegen die Sowjetunion“ interessiert war (dies., S. 45). Die Kooperation mit der kriegsgeschichtlichen Abteilung der amerikanischen Armee eröffnete Halder die unerwartete Chance, die Geschichtsschreibung zum Zweiten Weltkrieg und zur Rolle der Wehrmacht in die aus seiner Sicht „,richtigen Bahnen zu lenken“ (dies., S. 49). Wie, neben anderen Beiträger_innen, Ronald Smelser und Edward J. Davies II zeigen, schrieben aber auch vor allem amerikanische Autoren am verklärend-heroisierenden Narrativ mit. In den USA entstand eine regelrechte „Subkultur“ aus „Militärenthusiasten“, „Modellbauern“, Sammlern von „Militaria und NS-Devotionalien“" bis hin zu Neo-Nazis, die mit Vorliebe auf Bücher bestimmter Autoren zurückgreifen, die für diesen Personenkreis mittlerweile den Status von Gurus einnehmen, weil sie genau das liefern, was gewünscht wird: das „Hohe Lied von Tapferkeit und Opfergang deutscher Soldaten an der Ostfront, genialen Militäroperationen und überlegener Militärtechnik“" (S. 64).

Andere Aufsätze behandeln die beiden Wehrmachtsaustellungen (Hannes Heer, der die erste, auch die Rolle der ,kleinen“ Soldaten thematisierende Ausstellung (mit)verantwortet hatte, im persönlichen Rückblick), den Stalingrad-Mythos in der deutschen Nachkriegsliteratur (Jörg Füllgrabe), den deutschen Kriegsfilm der 1950er Jahre (Marcus Stiglegger), den um „Apologie und Erlösung“ (S. 168) bemühten ZDF-Dreiteiler „Unsere Mütter, unsere Väter“ (Volker Benkert) und das „Deutsche Panzermuseum Munster“ (Ralf Raths). Breiten Raum nimmt die Fabrikation und Tradierung der Heldenkulte und -legenden um „Jagdflieger-Asse“ (Jens Wehner), U-Boot-Kommandanten wie Günther Prien (Thomas Riederer), Otto Kretschmer oder Joachim Schepke (Daniel Uziel) und die Kavallerieverbände der Waffen-SS (Henning Pieper) ein. Besonders verdienstvoll ist, dass in der Forschung bislang eher vernachlässigte Themen wie die Kriegsromanheftreihe „Blaue Jungs“ über die Matrosen der Kriegsmarine (Gerhard Wiechmann) als Pendant zum „Landser“ oder die politische Indienstnahme der Militärmusik (Heike Frey) ebenfalls in den Band aufgenommen wurden.

Den Abschluss bildet ein Beitrag über die „Division ,Brandenburg““, ein aus „minderheitendeutschen Kriegsfreiwilligen aus ganz Europa und dem Nahen Osten“, Auslands- und Reichsdeutschen sowie Österreichern zusammengesetzter Verband (Johannes Kramer, S. 331), der beispielsweise als „Personalressource für Sabotage- und Insurrektionseinsätze“ - heute ,unconventional warfare“ genannt - diente (S. 332). An diesem Beispiel wird die politische Brisanz gewisser Formen der ,Traditionspflege" besonders deutlich, stellten doch die Autoren einer einschlägigen Publikation zu den ,legendären ,Brandenburgern““ - darunter der zeitweilige Kommandeur des Kommandos Spezialkräfte, Reinhard Günzel, und der Gründer und erste Kommandeur der GSG 9, Ulrich K. Wegener - eine ,direkte Kontinuität zwischen dem Sonderverband der Wehrmacht und den gegenwärtigen deutschen 
Spezialeinheiten“ her (S. 334). Will man angesichts der teilweise niederschmetternden Befunde nicht in Resignation verfallen, bleibt kaum etwas anderes übrig, als die hartnäckig gehegten und gepflegten Legenden um die „saubere“ Wehrmacht, die „,besten Soldaten der Welt“, die „Distanz“ zum NS-Regime oder die Waffen-SS als eine „Art Vorläufer“ der heutigen EU ebenso hartnäckig und beharrlich zu widerlegen. Der hervorragende Sammelband liefert zu diesem Zweck bestens geeignete Informationen und Überlegungen.

Funding Open Access funding enabled and organized by Projekt DEAL.

Open Access Dieser Artikel wird unter der Creative Commons Namensnennung 4.0 International Lizenz veröffentlicht, welche die Nutzung, Vervielfältigung, Bearbeitung, Verbreitung und Wiedergabe in jeglichem Medium und Format erlaubt, sofern Sie den/die ursprünglichen Autor(en) und die Quelle ordnungsgemäß nennen, einen Link zur Creative Commons Lizenz beifügen und angeben, ob Änderungen vorgenommen wurden.

Die in diesem Artikel enthaltenen Bilder und sonstiges Drittmaterial unterliegen ebenfalls der genannten Creative Commons Lizenz, sofern sich aus der Abbildungslegende nichts anderes ergibt. Sofern das betreffende Material nicht unter der genannten Creative Commons Lizenz steht und die betreffende Handlung nicht nach gesetzlichen Vorschriften erlaubt ist, ist für die oben aufgeführten Weiterverwendungen des Materials die Einwilligung des jeweiligen Rechteinhabers einzuholen.

Weitere Details zur Lizenz entnehmen Sie bitte der Lizenzinformation auf http://creativecommons.org/ licenses/by/4.0/deed.de.

\section{Ganzenmüller, Jörg (Hrsg.): Jüdisches Leben in Deutschland und Europa nach der Shoah. Neubeginn - Konsolidierung - Ausgrenzung, 296 S., Böhlau, Köln 2020.}

\section{Klaus-Peter Friedrich}

Angenommen: 30. Juni 2021 / Online publiziert: 9. Juli 2021

(C) Gesellschaft zur wissenschaftlichen Förderung politischer Literatur e.V. and the Author(s) 2021

Wie Jörg Ganzenmüller im Titel seiner Einleitung formuliert, geht es in diesem Sammelband um jüdisches Leben nach der Schoah aus einer ,erfahrungsgeschichtlichen und transnationalen Perspektive“ (S. 9). Hintergrund ist die Entwicklung in

\footnotetext{
Klaus-Peter Friedrich $(\bowtie)$

Marburg, Deutschland

E-Mail: klaus-peter.friedrich@gmx.de
} 